\title{
Wine analysis to check quality and authenticity by fully-automated ${ }^{1} \mathrm{H}-\mathrm{NMR}$
}

\author{
Manfred Spraul, Markus Link, Hartmut Schaefer, Fang Fang, and Birk Schuetz \\ Bruker BioSpin GmbH, Rheinstetten 76287, Germany
}

\begin{abstract}
Fully-automated high resolution ${ }^{1}$ H-NMR spectroscopy offers unique screening capabilities for food quality and safety by combining non-targeted and targeted screening in one analysis (15-20 min from acquisition to report). The advantage of high resolution ${ }^{1} \mathrm{H}-\mathrm{NMR}$ is its absolute reproducibility and transferability from laboratory to laboratory, which is not equaled by any other method currently used in food analysis. NMR reproducibility allows statistical investigations e.g. for detection of variety, geographical origin and adulterations, where smallest changes of many ingredients at the same time must be recorded. Reproducibility and transferability of the solutions shown are user-, instrument- and laboratory-independent. Sample preparation, measurement and processing are based on strict standard operation procedures which are substantial for this fully automated solution. The non-targeted approach to the data allows detecting even unknown deviations, if they are visible in the ${ }^{1} \mathrm{H}-\mathrm{NMR}$ spectra of e.g. fruit juice, wine or honey. The same data acquired in high-throughput mode are also subjected to quantification of multiple compounds. This ${ }^{1} \mathrm{H}-\mathrm{NMR}$ methodology will shortly be introduced, then results on wine will be presented and the advantages of the solutions shown. The method has been proven on juice, honey and wine, where so far unknown frauds could be detected, while at the same time generating targeted parameters are obtained.
\end{abstract}

\section{Introduction}

High Resolution NMR Spectroscopy for long time was considered a tool to allow structure elucidation of pure compounds. With the advent of Metabolomics, it could be shown, that ${ }^{1} \mathrm{H}-\mathrm{NMR}$ is a general tool in complex mixture analysis and also offers unique screening capabilities for food quality and safety by combining non-targeted and targeted screening in one measurement. The objective is to demonstrate, that due to its extreme reproducibility and transferability NMR can detect smallest changes in concentrations of many components in a mixture, which is best monitored by statistical evaluation however also delivers reliable quantification results. NMR has been proven to be fully quantitative over a wide concentration range and can work with a single calibration sample for all compounds to be quantified, therefore no need for calibration of each individual compound exists.

\section{Materials and method}

A $400 \mathrm{MHz}$ spectrometer with proton optimized detection and automatic sample changer forms the basis of the NMR screening procedure. Strict SOP's have been established for minimized sample preparation, storage, measurement and processing for each matrix to be screened.

For wine, the sample preparation is performed as follows: $540 \mathrm{ml}$ of wine are mixed with $60 \mathrm{ml}$ of $\mathrm{D}_{2} \mathrm{O} /$ Buffer solution. The buffer, 1.5 molar Potassium hydrogen phosphate in $\mathrm{D}_{2} \mathrm{O}$, is used deliver the reference signal for locking and to stabilize the $\mathrm{pH}$. The $\mathrm{pH}$ is fine-adjusted using a semi-automatic small scale system $(\mathrm{BTpH}$, Bruker
BioSpin GmbH Germany). NMR measurement is performed at $300 \mathrm{~K}$ using 32 scans and suppressing water and ethanol signals by selective shaped pulses. In addition a 2D J-Resolved experiment is run to allow safe identification and quantification. Spectra obtained are processed under full automation.

Per registered spectrum, different interpretation routines are performed. Each interpretation of data is carried out by univariate and multivariate statistical analysis to reference models based on authentic samples (non-targeted mode). The system is calibrated with a reference database containing spectra of representative samples covering the natural variability of the examined sample type. The quality of interpretation depends on representativeness and the number of reference spectra. The databases for food analysis are built up using certified authentic samples of e.g. juices, wines or honey from industrial processes. All spectra have to be acquired under identical conditions to ensure comparability. For each sample a report including origin, variety and condition of e.g. processed fruits, in addition this applied technology is available to guarantee traceability.

From the large range of available multivariate statistical methods applied in context of metabolomics data analysis [1,2] different possibilities of comparison can be used. A multitude of NMR spectral parameters can be entered into mathematical models. The combination of methods can improve sample characterisation and in some cases final judgement. Individual components are identified and quantified using a knowledge base and information from a reference compound NMR spectral base (targeted mode). 


\section{Results and discussion}

\subsection{Juice analysis}

The SGF-Profiling ${ }^{\mathrm{TM}}$ for fruit juice, launched in 2009, represents a heterogeneous collection of cascading statistical models which can be applied consecutively to one single spectrum, see Fig. 1, such as specific models for multi fruit type separation, fruit type differentiation between citrus varieties (e.g. citrus sinensis and citrus reticulata), differentiation of product categories (e.g. orange juice and orange juice made from concentrate), or characterisation of compositional differences for two groups of similar products (e.g. apple juice concentrates from Poland and China) [3,4]. Fig. 1 left side shows a quantile plot including all spectra that are part of a model for authenticity. The black curve representing the current sample is superimposed to the superposition of all model spectra and should in all parts fall within the colored envelope, representing the range of normal variation to be compliant. The left part shows the univariate verification, where each data point of the sample spectrum is compared to the normal variation in the model at the same spectral position. Figure 1 right side shows a plot a multivariate verification, where intensities of one point are compared to the intensity of all data points in the spectrum. Both procedures allow to identify under full automation if there are any outlying signals in the current sample spectrum and also list the chemical shift of such spectral regions.

For better performance of compound identification and quantification in case of signal overlap, a rapid 2D J-Resolved spectrum [5] is recorded as part of the standard routine (Fig. 2). By vertically aligning the coupling information, overlap is drastically reduced and unambiguous identification and quantification is achieved.

\subsection{Extension to wine analysis}

Having established the statistical and quantification methodology, it is possible to expose the technology described for fruit juices to other food material by exchanging the underlying spectral database from juice for example to wine. The knowledge base for quantification in this case has to be modified as well to represent chemical shift variation of the compounds to be quantified, as they are observed in a different matrix, which can also produce different signal overlay problems. Applying the statistical technology to wines allows e.g. to differentiate variety, geographical origin and vintage.
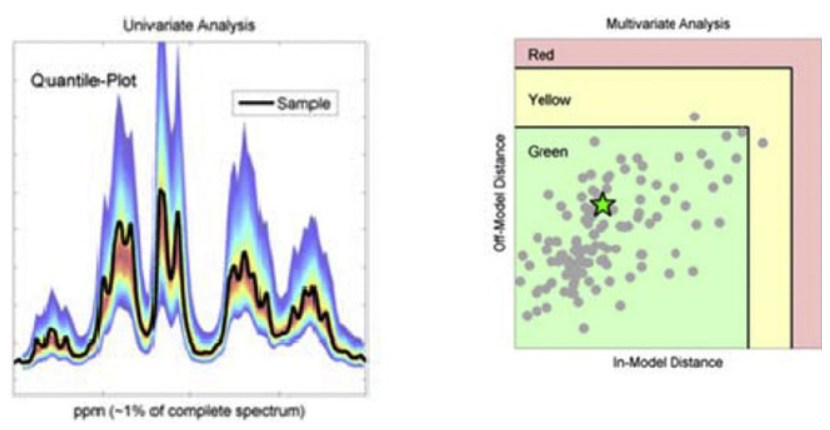

Figure 1. Univariate analysis vs. multivariate analysis in NMR based food screening.

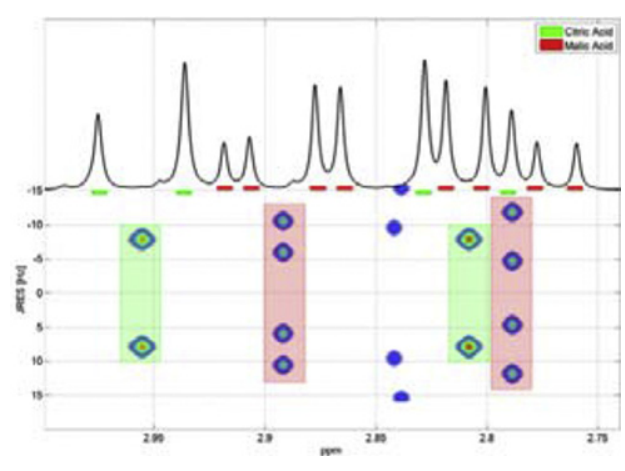

Figure 2. Rapid 2D J-Resolved spectrum recorded during the automatic measurement procedure shows a region of the 1D (NOESY-Presat) and 2D (JRES) spectra of a pear juice. Signals of Citric Acid (green) and Malic Acid (red) are separated in the 2D-spectrum.

Within 3 years the analysis could be expanded from 25 to more than 100 parameters offering a major increase in parameter density with regard to quantification and statistical analysis. Several of those parameters are either not available with conventional analysis or need multiple conventional methods to run. Multiple conventional methods also mean different sample preparation procedures are needed, such increasing the cost of the analysis substantially. Therefore the Wine-Screening by NMR produces results at low cost per sample and per parameter. Since quality and authenticity are ever increasing in importance for the consumer due to a large number of frauds being published, the NMR method comes at the right moment.

The statistical models developed on a base of 13.000 spectra now contain 5 relevant countries and 7 relevant regions e.g. in France (Bordeaux), Italy (Toscana, Sicilia, Puglia and Piemonte) and Spain (La Rioja \& Ribera del Duero), that can be verified. Classification analysis includes 22 grape varieties and validation of white wine vintages (2011-2014). A rapid detection of water addition for selected grape varieties is recently available in addition.

For this $400 \mathrm{MHz}$ push-button system, which can also perform juice and honey screening, standard operation procedures for wine analysis have been defined and optimized, that also include sample preparation which can be done manually or by a robotic system (including Bruker's $\mathrm{BTpH}$ system). Due to the highest reproducibility of NMR, statistical analysis is extremely powerful, is lab-, user- and instrument-independent and allowing detecting smallest changes of many parameters at a time. This enables for example the determination of geographical origin of wines first of all on a country wide basis, but also for sub-areas within a country. For Bordeaux wines a differentiation is given. The full potential is shown in Fig. 3.

Besides the geographical origin, also the grape variety is an important parameter, defining also the price of the wine. Worldwide variety models have been developed for a multitude of red (Fig. 4) and white wines (Fig. 5). For wines from Germany and Austria further varieties are parts of the analysis. In the same way vintage can be differentiated for German and Austrian wines.

The verification part of WineScreener result contains the function of being able to detect all types of NMR 
Result: Declared region Bordeaux is consistent with classification result.

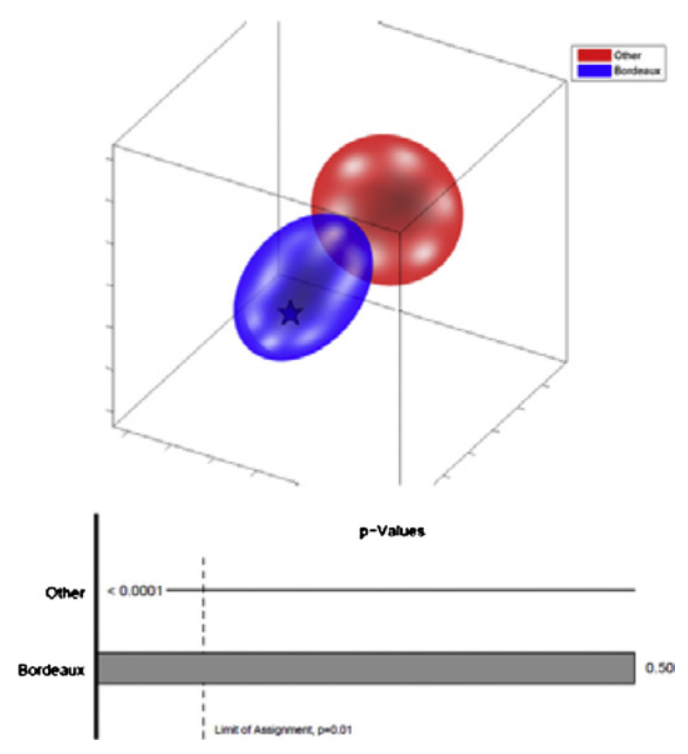

Figure 3. Result of a Bordeaux wine presented in graphical form with probability values.

visible deviations, be they known or unknown and therefore contribute not only to product quality, but also to product safety and controlling labelling (Fig. 6), wine content (= addition of water; Fig. 7) and adulteration.

Quantification of compounds in wine delivers 56 values in a report, which is generated automatically. For each value the distribution of concentrations in the corresponding authentic model is given. This allows drawing conclusions also from quantitative parameters not defined for example in the OIV (Organisation International Du Vin) and such produces additional criteria for quality of a wine. Figure 8 shows an excerpt from the quantification part of the wine report that is generated automatically after every measurement. LOQ's are also given besides the absolute concentration values for each compound. In the distribution curves on the right side of the table, the black bar indicates the actual sample on top of the NMR derived concentration profile.

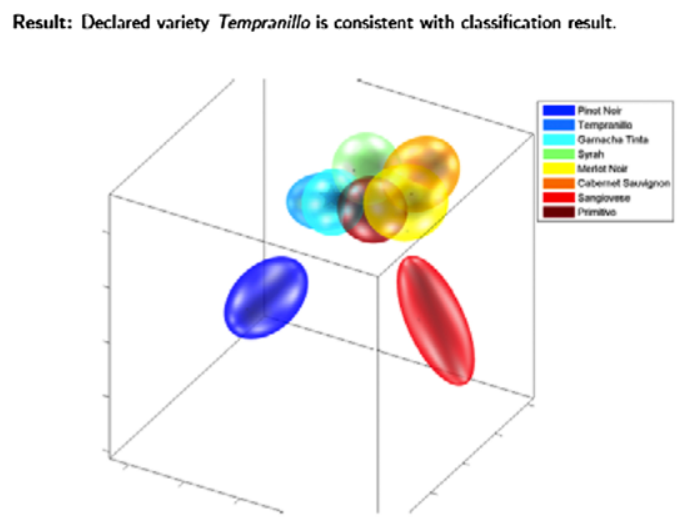

Result: Declared variety Silvaner Grüner is consistent with classification result.

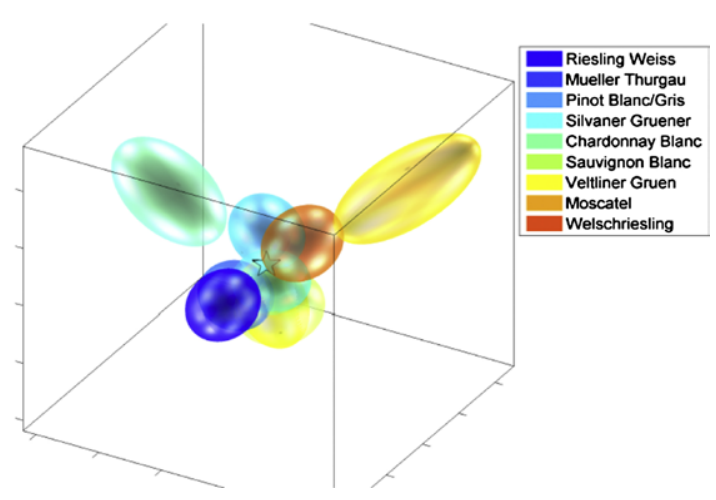

Figure 5. Example of international white wine variety models.

The example given indicates the potential of NMR in food quality and safety, being transferable from one material to another applying the corresponding normal sets. Investigations have also been made on honey.

\section{Conclusions}

The Wine-Profiling explained represents a NMR-based high throughput screening solution for wine. The combination of statistical modelling with molecule specific signal analysis makes it a powerful tool for food quality assessment. Specific quality aspects, wine content analysis (= addition of water) and adulterations can be identified by applying a number of statistical tests on the same spectrum. Fruit and product type differentiation, adulteration by sugar or acid addition, geographical origin and fruit mixtures are predictable as well as grape variety, vintage and geographical origin. Moreover, a list of substances being present in wine is quantified. The application of NMR based Wine-Profiling in routine controls are enhancing the control density and the amount of detected quality deviations. The pre-selection carried out allows choosing well-targeted conventional analysis for a sample. In many cases, it can save higher analytical costs. Therefore WineProfiling is useful and extremely economic in cases where

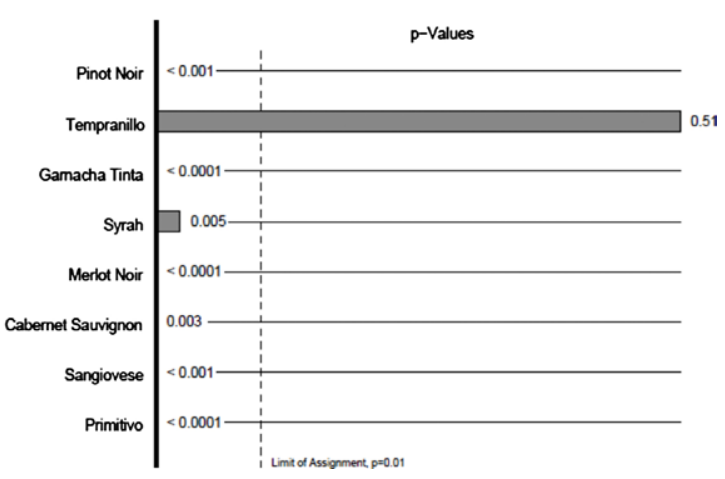

Figure 4. Example of international red wine variety models. 


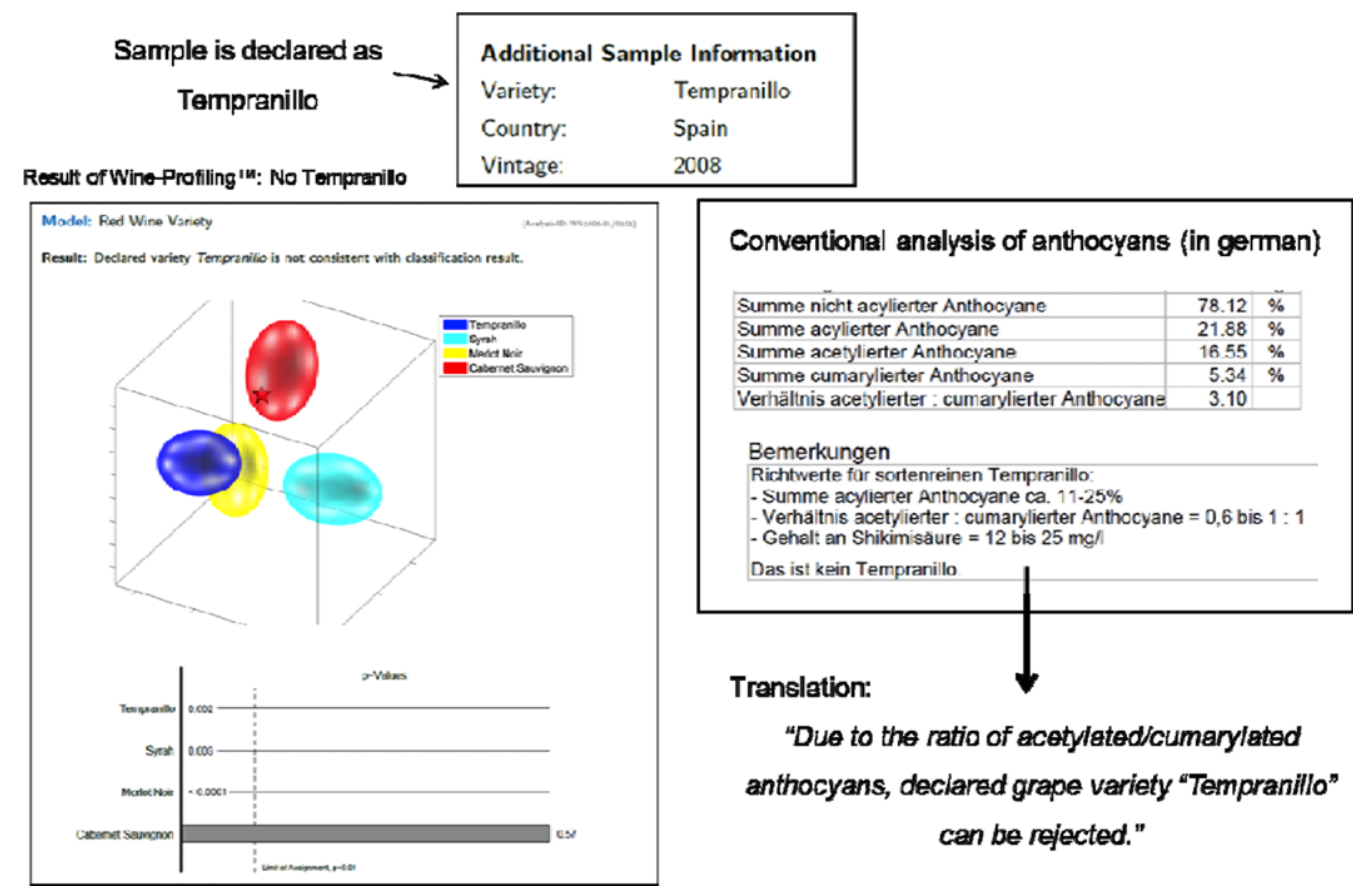

Figure 6. Example of wrong labelling.

Sample under investigation: Cabemet Sauvignon

- Wine Profiling:

"significant indication for an addlition of water"

- Isotope analysis confilmed addition of water

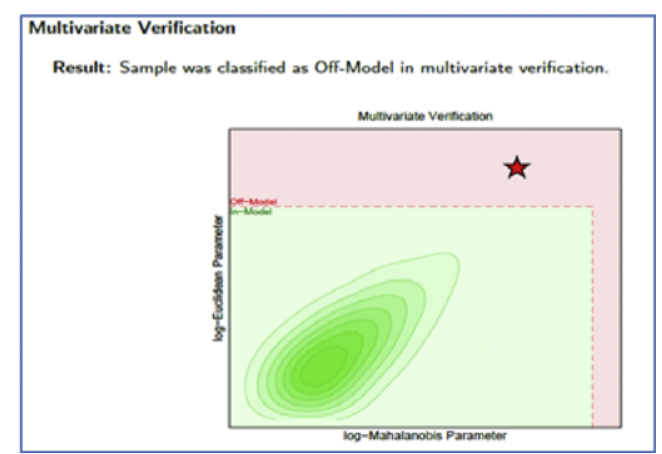

Figure 7. Example of wine content analysis (addition of water).

Targeted Analysis

In the following tables the results of the quantitative analysis are given. Parameters labelled with - are
calculated parameters. Please refer to the additional remarks for quantified pararameters. flags and reference values on page 8 . The displayed distributions of the Wine-Profiling 1 NMR reference

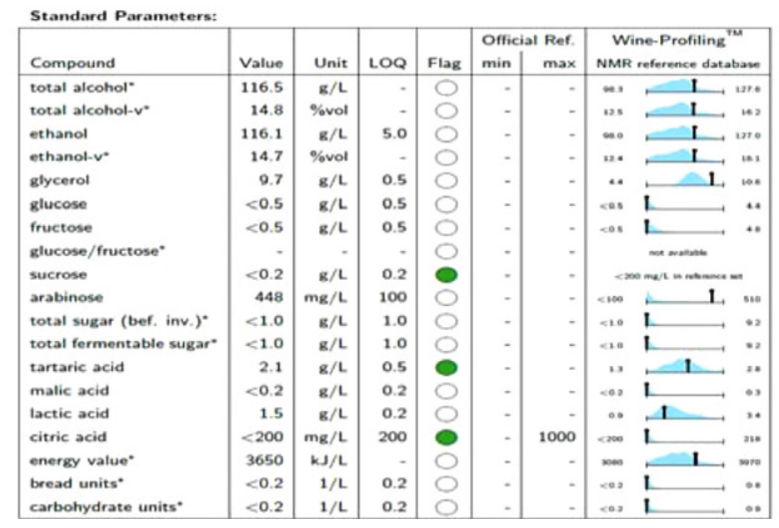

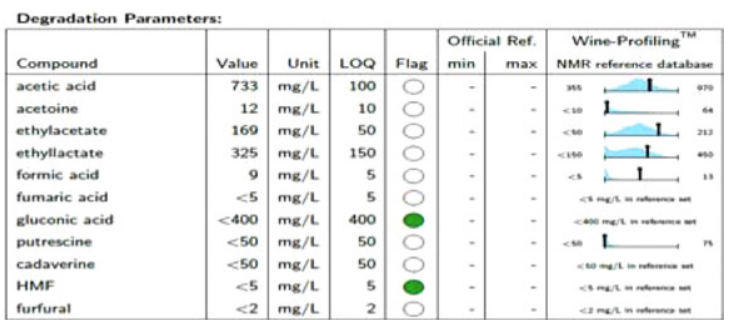

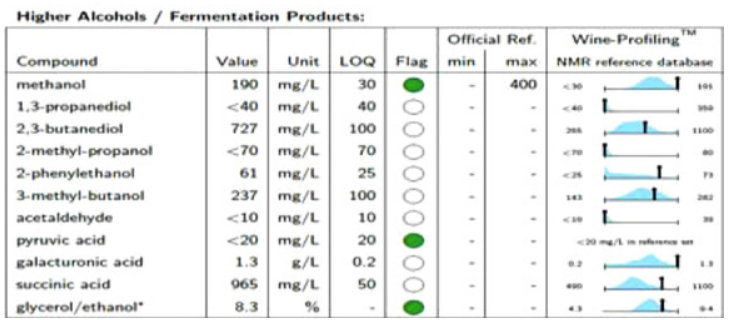

Figure 8. Excerpt of an identification/quantification table of a WineScreener report. 
samples need a very fast analytical answer and where a large number of samples should be tested and can be transferred to other food materials as shown [6]. Wine-Profiling is lab-, user- and instrument-independent.

SGF International e.V., Nieder-Olm, Germany; Winespin-Analytics, Aspisheim, Germany; Eurofins Analytics, Nantes, France; lfz Klosterneuburg, Klosterneuburg, Austria.

\section{References}

[1] B.G.M. Vandeginste et al. (1998). Handbook of Chemometrics and Qualimetrics. 2 Elsevier.
[2] T. Hastie et al. (2001). The Elements of Statistical Learning - Data Mining, Inference and Prediction. Springer.

[3] H.-J. Hofsommer (1999). Fruit Processing. New Analytical Techniques for Judging the Authenticity of Fruit Juices - an overview, p. 471-479

[4] S. Koswig (2006). Fruit Processing, determination of foreign fruit types and fruit varieties - Analysis. Evaluation and Practical Problems. p. 401-407

[5] S. Braun et al. (1998). 150 and More Basic NMR Experiments. Wiley- $\mathrm{VCH}$

[6] Lachenmeier et al. (2009). Journal of Agriculture and Food Chem. 57, p. 7194-7199 